EPJ Web of Conferences 19, 03008 (2012)

DOI: $10.1051 /$ epjconf/20121903008

(C) Owned by the authors, published by EDP Sciences, 2012

\title{
Looking for tidal streams around Galactic globular clusters
}

\author{
J.A. Carballo-Bello ${ }^{1,2, a}$ and D. Martínez-Delgado ${ }^{3}$ \\ ${ }^{1}$ Instituto de Astrofísica de Canarias (IAC), vía Láctea s/n, 38205 La Laguna, Spain \\ ${ }^{2}$ Departamento de Astrofísica, Universidad de La Laguna, 38205 La Laguna, Spain \\ ${ }^{3}$ Max-Planck-Institut für Astronomie, 69117 Heidelberg, Germany
}

\begin{abstract}
The picture of building the Milky Way halo from merging protogalactic fragments is considered the local manifestation of the hierarchical galaxy formation process. In this scenario, some observational evidences have suggested that the outer young Galactic halo globular cluster population might be associated (or even the nuclei) to tidal disrupted dwarf spheroidals, now extinct galaxies. If this hypothesis is true, these systems might be surrounded by a distinct and still detectable stellar population. We have carried out a systematic observation of Galactic globulars covering the galactocentric distance range $10<\mathrm{R}_{\mathrm{GC}}<40 \mathrm{kpc}$ in both hemispheres. We have used wide field instruments both in La Palma and in La Silla observatories to obtain deep photometry of wide areas around these globulars to unveil the possible remnants of their progenitor dwarf galaxies.
\end{abstract}

\section{INTRODUCTION}

Globular cluster (GC) systems contain valuable information about the formation process of the host galaxy. In the case of the Milky Way, the existence of two subcomponents in the GC system was interpreted as an evidence supporting the hierarchical galaxy formation scenario proposed by Zinn (1993). The outer halo clusters $\left(\mathrm{R}_{\mathrm{GC}} \geq 15 \mathrm{kpc}\right)$ were probably formed in small fragments subsequently accreted by the Galaxy and it is possible that many of them are the remnant core of the disrupted progenitor as suggested by van den Bergh and Mackey (2004), whereas the inner clusters were formed "in situ". More recent analysis of the relative ages of GCs by Marín-Franch et al. (2009) also found two distinct and well-defined groups of clusters in the Milky Way for $\mathrm{R}_{\mathrm{GC}} \leq 20 \mathrm{kpc}$. On the one hand, an old population of clusters with small dispersion in relative ages of $\sim 0.6 \mathrm{Gyr}$, which is consistent with the initial collapse of a protogalaxy with a total mass similar to that of the Milky Way. On the other hand, they noted the existence of a second group of younger GCs with a clear age-metallicity relation that might be associated with the continuous accretion of the building blocks suggested by the $\Lambda \mathrm{CDM}$ simulations.

\section{OUR PROJECT}

If these GCs were formed in subsequently accreted stellar systems, these clusters might be still surrounded by the tidal streams generated by the disruption of their progenitor satellites. This idea has been probed by Bellazzini et al. (2003) with the detection of Sagittarius stream stars around several Galactic GCs as Pal 12 or NGC 4147 in the 2MASS data. In the case of Pal 12, wide-field photometry presented by Martínez-Delgado et al. (2002) has revealed the presence of a subjacent

ae-mail: jacb@iac.es

This is an Open Access article distributed under the terms of the Creative Commons Attribution-Noncommercial License 3.0, which permits unrestricted use, distribution, and reproduction in any noncommercial medium, provided the original work is properly cited. 


\section{EPJ Web of Conferences}
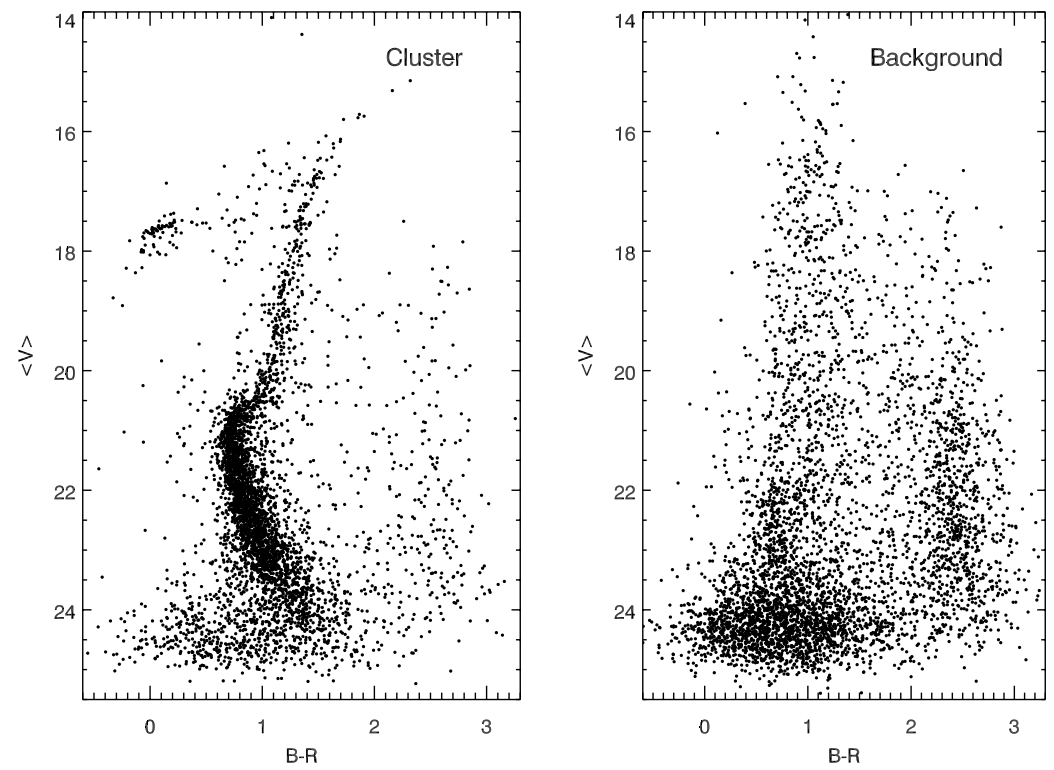

Figure 1. Colour-magnitude diagrams showing the content of the GC NGC 5634 (left) and its surroundings (right). We find a subjacent stellar population as a main-sequence below $\mathrm{V}=22$ around $\mathrm{B}-\mathrm{R} \sim 0.7$.

stellar population associated with the Sagittarius tidal stream with a compatible distance with that of the cluster. Following the same techniques, Martínez-Delgado et al. (2004) discovered features in the colour-magnitude diagrams of the surrounding areas of a sample of outer halo clusters. We have designed a systematic deep wide-field imaging of a sample of 25 Galactic globular clusters lying in the range $10<\mathrm{R}_{\mathrm{GC}}<40 \mathrm{kpc}$ and using the WFC+INT and WFI+ESO $2.2 \mathrm{~m}$ instruments.

Our photometry allows us to explore the outer regions of the clusters, obtaining the radial density profiles shown in Carballo-Bello et al. (2011) which are used to distinguish between cluster and background populations. Our results show that the projected limits of an important fraction of the GCs is beyond the previously known tidal radius. There exists around a third of our sample a subjacent stellar population, possibly associated with some of the known tidal streams (e.g. Sagittarius, Monoceros) or with an undetected low-surface brightness halo overdensity. Follow-up spectroscopy is required.

\section{References}

Bellazzini, M., Ibata, R., Ferraro, F. R., and Testa, V.: 2003, A\&A 405, 577

Carballo-Bello, J. A., Gieles, M., Sollima, A., Koposov, S., Martínez-Delgado, D., and Peñarrubia, J.: 2011, arXiv:1108.4018

Marín-Franch, A., Aparicio, A., Piotto, G., Rosenberg, A., Chaboyer, B., Sarajedini, A., Siegel, M., Anderson, J., Bedin, L. R., Dotter, A., Hempel, M., King, I., Majewski, S., Milone, A. P., Paust, N., and Reid, I. N.: 2009, ApJ 694, 1498

Martínez-Delgado, D., Dinescu, D. I., Zinn, R., Tutsoff, A., Côté, P., and Boyarchuck, A.: 2004, in F. Prada, D. Martinez Delgado, \& T. J. Mahoney (ed.), Satellites and Tidal Streams, Vol. 327 of Astronomical Society of the Pacific Conference Series, pp $255-+$

Martínez-Delgado, D., Zinn, R., Carrera, R., and Gallart, C.: 2002, ApJL 573, L19

van den Bergh, S. and Mackey, A. D.: 2004, MNRAS 354, 713

Zinn, R.: 1993, in G. H. Smith \& J. P. Brodie (ed.), The Globular Cluster-Galaxy Connection, Vol. 48 of Astronomical Society of the Pacific Conference Series, pp $38-+$ 